\title{
Infecciones invasivas por serotipos no vacunales de Streptococcus pneumoniae: una amenaza creciente
}

\author{
Lorena Rodríguez-Muñoz ${ }^{1 *}$, Fortino Solórzano-Santos², Edgar A. Flores-Reyes ${ }^{1}$, Juana Escalante-López ${ }^{1}$, \\ Dora E. Rodríguez-Balderas ${ }^{1}$, Gabriela Echániz-Avilés ${ }^{3}$ y M. Noemi Carnalla-Barajas ${ }^{3}$ \\ ${ }^{1}$ Hospital Del Niño Dr. Federico Gómez Santos, Saltillo, Coah.; ${ }^{2}$ Hospital Infantil de México Federico Gómez, Ciudad de México; ${ }^{3}$ Instituto Nacional \\ de Salud Pública, Cuernavaca, Mor. México
}

\begin{abstract}
Resumen
Introducción: En México, cuando se inició la aplicación de la vacuna PCV13 (neumocócica conjugada), se cubría el 70.6\% de los serotipos causantes de enfermedad invasiva por neumococo en menores de 5 años. Después de varios años, los casos de enfermedad causada por los serotipos incluidos en la vacuna han disminuido; sin embargo, se ha producido un reemplazo por los serotipos no incluidos en la vacuna. Caso clínico: Se presentan tres casos de pacientes pediátricos que desarrollaron enfermedad invasiva por serotipos no incluidos en la PCV13: uno con meningitis y bacteriemia (serotipo 15C) y dos con neumonía, uno de ellos complicado con derrame (serotipo 35B). Los pacientes fueron atendidos en un hospital pediátrico en Saltillo, Coahuila, durante el periodo de 2015 a 2018. Conclusiones: Resulta alarmante que se presenten tres casos graves por serotipos de Streptococcus pneumoniae no incluidos en la PCV13 en un solo hospital pediátrico en el norte del país. Este es un fenómeno que esta sucediendo a escala nacional e internacional: un incremento de casos de enfermedad invasiva por serotipos de neumococo no incluidos en la vacuna utilizada actualmente.
\end{abstract}

Palabras clave: Streptococcus pneumoniae. Reemplazo de serotipos. Vacunas conjugadas. Meningitis. Neumonía complicada.

\section{Invasive infections by non-vaccine serotypes of Streptococcus pneumoniae: a growing threat}

\begin{abstract}
Background: In Mexico, $70.6 \%$ of serotypes causing invasive pneumococcal disease were covered since the application of the PCV13 vaccine in children under 5 years of age. After several years of immunization, cases of disease caused by the serotypes included in the vaccine have decreased. However, a replacement due to serotypes not included in the vaccine has been observed. Case report: Three cases of pediatric patients who developed invasive disease due to serotypes not included in PCV13 are described: one with meningitis and bacteremia (serotype 15C), and two with pneumonia, of which one complicated with effusion (serotype 35B). Patients were treated in a pediatric hospital in Saltillo, Coahuila, from 2015 to 2018. Conclusions: Three serious cases due to serotypes of Streptococcus pneumoniae not included in PCV13 were reported in a single pediatric hospital in a northern state of Mexico. This phenomenon is taking place nationwide and worldwide: an increase of cases of invasive disease due to pneumococcal serotypes not included in the vaccine currently used.
\end{abstract}

Key words: Streptococcus pneumoniae. Serotype replacement. Conjugate vaccines. Meningitis. Complicated pneumonia.

Correspondencia:

*Lorena Rodríguez-Muñoz

E-mail: dra.lorenardzm@gmail.com

DOI: $10.24875 /$ BMHIM. 19000046
Disponible en internet: 20-09-2019 Bol Med Hosp Infant Mex. 2019;76:281-286 www.bmhim.com

1665-1146/@ 2019. Hospital Infantil de México Federico Gómez, impreso por Permanyer México SA de CV, todos los derechos reservados. 


\section{Introducción}

Streptococcus pneumoniae es considerado como uno de los agentes bacterianos más frecuente en la edad pediátrica. Es causante de enfermedades invasivas como meningitis y sepsis, así como de neumonía adquirida en la comunidad, otitis media y artritis, entre otras. Una de las estrategias para el control de los casos graves ha sido la inclusión de la vacuna contra este microorganismo. La vacuna polisacárida de 23 serotipos no genera una adecuada respuesta inmunogénica en menores de 2 años. Por lo anterior, después de varios ensayos clínicos ${ }^{1,2}$, una vacuna conjugada proteína-polisacáridos contra 7 serotipos (PCV7) fue aprobada para su uso en los EE.UU. en febrero del año 2000. Esta vacuna demostró una adecuada respuesta inmune en los menores de 2 años con una cobertura del 70 al $88 \%$ de los serotipos causantes de enfermedad invasiva.

En el 2006, la vacuna PCV7 se empezó a aplicar en grupos especiales en México, y fue incorporada a la cartilla nacional de vacunación en 2008. En 2012 fue sustituida por la vacuna conjugada contra 13 serotipos (PCV13): serotipos 1, 3, 4, 5, 6A, 6B, 7F, 9V, 14, 18C, $19 \mathrm{~A}, 19 \mathrm{~F}$ y $23 \mathrm{~F}$. Esta vacuna se aplica actualmente con un esquema de $2+1$ dosis. Desde el inicio de su aplicación, la vacuna PCV13 cubría el $70.6 \%$ de los serotipos causantes de enfermedad invasiva en menores de 5 años ${ }^{3}$. Sin embargo, como un fenómeno anticipado, además de disminuir los casos de enfermedad invasora causada por los serotipos incluidos en la vacuna, se va produciendo un reemplazo de los serotipos que están colonizando la nasofaringe, por lo que se espera un incremento de casos de enfermedad por serotipos no vacunales. En este reporte se presentan tres casos de pacientes pediátricos atendidos en un hospital pediátrico en Saltillo, Coahuila, que desarrollaron enfermedad por serotipos no incluidos en la PCV13.

\section{Casos clínicos}

\section{Caso 1}

Paciente de sexo femenino de 2 años y 1 mes de edad, sin antecedentes de importancia para su padecimiento actual, con esquema nacional de vacunación completo para la edad, incluidas tres dosis de vacuna PCV13. Inició con sintomatología un día antes de su ingreso, al presentar una caída de su propia altura que le provocó una herida en el párpado superior, la cual fue tratada en una unidad de primeros auxilios mediante sutura, analgésico y antibiótico. Posteriormente, presentó hiporexia, somnolencia, vómito de contenido alimentario y fiebre no cuantificada controlada con medios físicos. La paciente continuó con somnolencia (alteración del estado de conciencia), por lo que al día siguiente fue llevada a consulta al hospital donde se le realizó control térmico. Presentó crisis convulsiva tónico-clónica generalizada de difícil control y tres crisis convulsivas focalizadas en la extremidad superior izquierda. Se le administraron benzodiazepinas, se impregnó con fenitoína sódica y se continuó con dosis de mantenimiento de $10 \mathrm{mg} / \mathrm{kg} / \mathrm{día}$. Asimismo, se realizó una tomografía simple de cráneo, la cual reportó parámetros normales. Los valores de los análisis clínicos fueron los siguientes: hemoglobina, $12.5 \mathrm{mg} / \mathrm{dl}$; leucocitos, $30,860 / \mathrm{mm}^{3}$; el $81 \%$ de neutrófilos y el $19 \%$ de linfocitos. La paciente presentó deterioro neurológico con escala de coma de Glasgow de 10 puntos y tensión arterial por encima del percentil 95 para la edad, por lo que se transfirió a la Unidad de Cuidados Intensivos Pediátricos. Se indicó vancomicina (60 mg/kg/día) y ceftriaxona (100 mg/kg/día). Continuó con deterioro neurológico, por lo que se realizó una intubación orotraqueal. Al día siguiente se realizó punción lumbar, y se obtuvo líquido cefalorraquídeo (LCR) transparente con los siguientes valores: leucocitos, $9 / \mu \mathrm{l}$; eritrocitos,

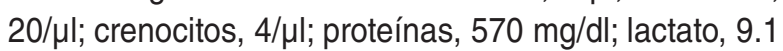
$\mathrm{mmol} / / \mathrm{l}$. Las pruebas de laboratorio identificaron el desarrollo de diplococos Gram positivos en el cultivo de LCR y hemocultivo, específicamente S. pneumoniae. A los 5 días de su ingreso, se realizó la extubación, y la paciente respondió a los estímulos táctil y doloroso. Por presentar crisis convulsiva focalizada en la extremidad superior izquierda y hemicara izquierda, se añadió levetiracetam (20 mg/kg/día) y ácido valproico (25 mg/kg/día). Después, la paciente presentó fasciculación de la comisura labial con respuesta a estimulo táctil y verbal y apertura ocular espontánea. A los 10 días de su ingreso presentó cinco picos febriles de $38.2^{\circ} \mathrm{C}$. Se realizó un cultivo de catéter central y tomografía computarizada (TC) simple de cráneo, que reportó incremento en la densidad y calibre del seno transverso derecho, que se extendía hasta el seno sagital superior, zonas hemorrágicas sobre el hemisferio cerebral izquierdo y edema cerebral de predominio izquierdo. Se retiró el catéter venoso por la persistencia de fiebre; a las $48 \mathrm{~h}$ se reportó infección por Pseudomonas aeruginosa, por lo que se suspendió la ceftriaxona y se agregó cefepima ( $150 \mathrm{mg} / \mathrm{kg} / \mathrm{d}$ ía), con lo que se controló la fiebre. Se continuó la vancomicina debido 
a la sospecha de infección asociada con el catéter. En total, recibió 21 días de manejo antibiótico.

Una vez completado el esquema de antibiótico, se realizó una TC, que mostró higroma subdural bilateral, prominencia en cisterna magna, permeabilidad del seno transverso y recto y lesión hipodensa frontal derecha no captante. Se egresó con anticomiciales y terapia de rehabilitación por hemiparesia facial derecha y disminución de fuerza muscular.

El Instituto Nacional de Salud Pública (INSP) reportó S. pneumoniae serotipo $15 \mathrm{C}$ en la muestra de cultivo de LCR y hemocultivo, resistente a la penicilina con una concentración mínima inhibitoria (CMI) de $4 \mu \mathrm{g} / \mathrm{ml}$.

\section{Caso 2}

Paciente de sexo femenino de 4 meses de edad, con una dosis de vacuna PCV13 aplicada a los 2 meses de edad. Inició su padecimiento 15 días antes de su ingreso con tos productiva y rinorrea hialina, por lo que acudió al médico particular, quien indicó tratamiento sintomático. Sin embargo, 3 días antes de su ingreso presentó agravamiento de los síntomas, ya que se agregaron datos de dificultad respiratoria a expensas de tiraje intercostal y polipnea, por lo que fue referida a esta unidad desde su clínica de primer nivel.

A su ingreso, la paciente se encontraba febril, hiporreactiva, pálida, con datos de dificultad respiratoria a expensas de aleteo nasal, tiraje intercostal, disociación toracoabdominal y retracción supraesternal, además de campos pulmonares hipoaireados con estertores. El resto de la exploración sin relevancia para el padecimiento. Se hospitalizó y se tomaron estudios de laboratorio y gabinete; la radiografía de tórax evidenció un patrón intersticial y alveolar con radiopacidad en el hemitórax izquierdo y el ápice derecho; la biometría hemática resultó con leucocitosis de $27,000 / \mathrm{mm}^{3}$ y predominio de linfocitos del $69.5 \%$. Se ingresó como neumonía adquirida en la comunidad de probable etiología bacteriana y se indicó esquema con ceftazidima y amikacina. La sintomatología se agravó y se realizó intubación endotraqueal. Como se presentó fiebre persistente $\left(39.8^{\circ} \mathrm{C}\right)$ de difícil control, se inició infusión de aminas por datos de choque séptico. Se valoró por el Servicio de Infectología Pediátrica, donde se indicó esquema antibiótico con ceftriaxona y clindamicina. Se tomó cultivo de aspirado bronquial al momento de la intubación. Después de $48 \mathrm{~h}$, se reportaron diplococos Gram positivos, posteriormente identificados como $S$. pneumoniae en el cultivo de secreción bronquial. La paciente fue extubada por mejoría inicial. Sin embargo, presentó sangrado por la cavidad oral, por lo que requirió reintubación y soporte ventilatorio. Al décimo día de internamiento, la paciente volvió a presentar fiebre y datos de dificultad respiratoria, por lo que se modificó el esquema de antibiótico (vancomicina y cefepima) por sospecha de neumonía asociada con el ventilador. La evolución fue tórpida: requirió manejo intensivo y presentó complicaciones asociadas con choque. La paciente falleció a los 30 días de estancia intrahospitalaria.

En el INSP se reportó $S$. pneumoniae serotipo 13 en el cultivo inicial de aspirado bronquial sensible a penicilina con una CMI de $2 \mu \mathrm{g} / \mathrm{ml}$.

\section{Caso 3}

Paciente de sexo femenino de 2 años y 9 meses de edad, previamente sana y sin antecedentes de importancia para su padecimiento actual. Esquema de vacunación completo con tres dosis de vacuna conjugada de neumococo. La paciente inició su padecimiento actual 10 días antes de su ingreso, con rinorrea hialina, fiebre no cuantificada, tos seca no emetizante, no cianozante ni en accesos, de predominio nocturno, por lo que fue llevada a atención médica. Recibió tratamiento con aciclovir, nimesulida, cetirizina y ambroxol con salbutamol por 4 días, sin mejoría. Cuatro días antes de su ingreso, la paciente presentó otorrea purulenta, razón por la cual fue llevada a otro facultativo, quien indicó manejo con cefixima, metamizol sódico y nebulizaciones con salbutamol e ipratropio. Se le proporcionó el tratamiento por 2 días, pero se detectó deterioro clínico, por lo que acudió de nuevo a valoración, indicándose dexametasona intramuscular. Un día antes de su ingreso, la paciente presentó quejido respiratorio, por lo cual fue llevada al hospital.

A su ingreso, la paciente se encontraba somnolienta, con periodos de irritabilidad, faringe hiperémica sin placas ni exudados, conductos auditivos externos sin otorrea, tórax con movimientos de amplexión y amplexación disminuidos, tiraje intercostal, disociación toracoabdominal y retracción xifoidea, campos pulmonares con estertores crepitantes diseminados en ambos hemitórax y con disminución del murmullo vesicular en la base izquierda, matidez en toda la base izquierda, sin transmisión de la voz y saturación del 74\% con aire ambiente. Se ingresó con diagnóstico de neumonía complicada con derrame pleural izquierdo. Se realizó biometría hemática que reportó $11.3 \mathrm{~g} / \mathrm{dl}$ de hemoglobina; $84,850 / \mathrm{mm}^{3}$ de leucocitos; $82 \%$ de neutrófilos; 9.5\% de linfocitos; $8.4 \%$ de monocitos; $382,000 / \mathrm{mm}^{3}$ plaquetas; PCR (proteína C reactiva) $>450$. 
Se realizó toracocentesis y colocación de sonda pleural izquierda. El análisis citoquímico del líquido pleural reportó líquido de aspecto turbio 4+, color amarillo, leucocitos $8,350 / \mathrm{mm}^{3}$, polimorfonucleares $68 \%$, linfocitos $26 \%$, eritrocitos $600 / \mathrm{mm}^{3}$, glucosa $10 \mathrm{mg} / \mathrm{dl}$, proteínas $300 \mathrm{mg} / \mathrm{dl}$, DHL (deshidrogenasa láctica) $22,500 \mathrm{U} / \mathrm{l}$, colesterol $63 \mathrm{mg} / \mathrm{dl}$, lactato $60 \mathrm{mmol} / \mathrm{l}$, glucosa sérica $175 \mathrm{mg} / \mathrm{dll}$, DHL sérica 2,137 U//, proteínas totales $6.4 \mathrm{~g} / \mathrm{dll}$ y albúmina $3 \mathrm{~g} / \mathrm{dl}$. Se prescribió doble esquema antibiótico con ceftriaxona y clindamicina. Al cuarto día, la paciente presentó evolución tórpida con derrame pleural en el hemitórax derecho. Se colocó sonda pleural, y se obtuvo nuevamente líquido pleural con características de empiema, y se escaló el manejo antibiótico con vancomicina, suspendiendo la clindamicina y manteniendo la ceftriaxona. Posteriormente, la paciente presentó buena evolución. Se logró retirar la sonda pleural izquierda a los 20 días de colocación y la derecha a los 16 días de colocación. La paciente recibió 21 días de tratamiento con ceftriaxona y vancomicina.

Luego de 1 mes de hospitalización, la paciente se egresó con ejercicios de terapia respiratoria y esteroide inhalado.

Se reportó crecimiento de S. pneumoniae en el cultivo del primer líquido pleural de hemitórax izquierdo. Se envió la cepa al INSP, donde se tipificó como serotipo 35B, sensible a penicilina con CIM de $1 \mu \mathrm{g} / \mathrm{ml}$. Del segundo líquido pleural de hemitórax derecho, el cultivo se reportó negativo.

\section{Discusión}

Las vacunas conjugadas actualmente disponibles solo cubren 10 o 13 de los más de 96 serotipos de S. pneumoniae. Inicialmente, se consideraba la posibilidad de reemplazo de los serotipos contenidos en la vacuna, pero no podía precisarse si el efecto se vería a corto o largo plazo. Actualmente, se ha comprobado este fenómeno en varios países ${ }^{4}$. El reemplazo de serotipos no incluidos en la PCV7 se observó a través de grandes sistemas de vigilancia por el aumento en la incidencia de la enfermedad debido a estos serotipos ${ }^{5}$. Para evaluar la epidemiología de S. pneumoniae se examinan los serotipos asociados con enfermedad invasiva temporalmente con el tipo de vacuna utilizado.

En este reporte se presentan tres casos de pacientes pediátricos, uno con meningitis y bacteriemia y dos con neumonía, cuya etiología fue $S$. pneumoniae de serotipos no incluidos en la vacuna conjugada PCV13.
En el primer caso, la paciente había recibido tres dosis de la vacuna PCV13. A pesar de ello, desarrolló meningitis y bacteriemia por $S$. pneumoniae serotipo 15C, con una elevada resistencia a penicilina. Este serotipo se encuentra dentro de los primeros cinco serotipos causantes de enfermedad invasiva no incluidos en la vacuna PCV13, de acuerdo con un reporte global $^{6}$. En algunos países, particularmente en Europa, es uno de los pocos serotipos resistentes a ertape$n^{7}$. En México, el grupo 15A/B/C, junto con el 35A/ $B / C / F$, son los serotipos no incluidos en la vacuna más frecuentes en población no vacunada ${ }^{8}$, y el grupo 15A/ $\mathrm{B} / \mathrm{C}$, el más frecuente en pacientes menores de 60 días ${ }^{9}$.

El segundo caso correspondió a una paciente que, por su edad, solo había recibido una dosis de PCV13. Inicialmente, desarrolló una neumonía comunitaria por S. pneumoniae serotipo 13. Durante su estancia hospitalaria, la paciente presentó neumonía asociada a la ventilación mecánica, complicación que contribuyó a su fallecimiento. En diferentes reportes, el serotipo 13 se ha aislado con baja frecuencia como causante de otitis media, particularmente en algunas comunidades de España ${ }^{10}$.

Como tercer caso se presentó una paciente con neumonía complicada con empiema bilateral. A pesar de ser inmunizada adecuadamente, se encontró el serotipo 35B en esta paciente, uno de los serotipos no vacunales más frecuentemente encontrados en México, como se mencionó previamente. En Massachusetts, se considera un serotipo de baja capacidad invasiva ${ }^{11}$.

Balsells, et al. ${ }^{6}$ realizaron un metaanálisis en el que se incluyeron 20 estudios de 24 países que habían introducido la PCV10 o PCV13. Se encontró que el $42.2 \%$ de los casos a escala global fueron episodios de enfermedad invasiva por serotipos no incluidos en la PCV13. Sin embargo, se observaron diferencias significativas de acuerdo con la región: el $57.8 \%$ en América del Norte, el $71.9 \%$ en Europa, el $45.9 \%$ en el Pacífico oeste, el $\mathbf{2 8 . 5 \%}$ en Latinoamérica, el $\mathbf{4 2 . 7 \%}$ en un país africano y el $9.2 \%$ en un país del este del mediterráneo. Los serotipos predominantes fueron los siguientes: 22F, 12F, 33F, 24F, 15C, 15B, 23B, 10A y 38. En este estudio, la información incluida sobre México representó los aislamientos de enfermedad invasiva en el año 2012. Estos aislamientos fueron concentrados por la red SIREVA (Sistema Regional de Vacunas), coordinada por el INSP y el Instituto de Diagnóstico y Referencia Epidemiológico. Se incluyó la información de todos los países en Latinoamérica (105 aislamientos), de los cuales, el $66.7 \%$ correspondió a serotipos 
incluidos en la PCV13 (19A, 19F, 3, 1, 23F, 6B, 6A, 9V, $7 F, 18 C$ y 14$)$ y el $33.3 \%$ a serotipos no incluidos en la vacuna $(35 \mathrm{~B}, 15 \mathrm{~B}, 23 \mathrm{~B}, 10 \mathrm{~A}, 6 \mathrm{C}, 11 \mathrm{~A}, 10 \mathrm{~F}, 7 \mathrm{C}, 12 \mathrm{~F}$, 22F, 15C, 20, 23A).

Para el año 2017, el Grupo Interdisciplinario para el Estudio de Enfermedades Bacterianas Prevenibles por Vacunación (GIVEBPVac), que solamente concentra los datos de México en el INSP, procesó 134 aislamientos, de los cuales el $44 \%$ fueron provenientes de casos de enfermedades invasivas (meningitis y septicemia) y el $55.3 \%$ correspondió a serotipos no incluidos en la vacuna. Los serotipos de reemplazo que se encontraron con mayor frecuencia fueron el 15C, 23A, 23B, 6C, $11 \mathrm{~A}$ y $35 \mathrm{~B}^{12}$. Es conveniente resaltar que el incremento en frecuencia de los serotipos no incluidos en la vacuna es relativo al disminuir los serotipos incluidos en la vacuna PCV13. Sin embargo, es evidente que actualmente existe un predominio de los serotipos no incluidos en la vacuna.

La inmunización con vacunas conjugadas también ha modificado la resistencia a antimicrobianos. Por ejemplo, en Corea, disminuyó la resistencia a penicilina de las cepas incluidas en la PCV13: del $80.8 \%$ en 2010 al $21.1 \%$ en 2014. Por el contrario, la resistencia de las cepas no incluidas en la vacuna incrementó del 19.2\% en 2010 al $78.9 \%$ en 2014 . Los serotipos principales que se encontraron fueron $23 \mathrm{~A}, 15 \mathrm{~B} / \mathrm{C}, 6 \mathrm{C}$ y $10 \mathrm{~A}^{13}$. Solamente uno de los casos presentados mostró el serotipo $15 \mathrm{C}$ resistente a penicilina (CIM $4 \mu \mathrm{g} / \mathrm{ml}$ ).

En un estudio de 15 años (1999-2014), en Dallas ${ }^{14}$, se encontró una disminución de la resistencia a penicilina en casos de meningitis: del $53.9 \%$ en la era prevacunal al $35.3 \%$ en la era post-PCV13. Sin embargo, estos resultados contrastaron con el incremento de la resistencia a penicilina en infecciones fuera del sistema nervioso central del 0 al 8.2\%. La resistencia a cefotaxima no mostró diferencias significativas para cualquier punto de corte. Los serotipos más comunes no incluidos en la PCV13 fueron el 23B, 6C, 23A y 12.

Es importante el hallazgo de tres casos graves por serotipos de S. pneumoniae no incluidos en la PCV13 en un solo hospital pediátrico del norte del país, lo que resulta concordante con el fenómeno que se está viviendo en México. Sobre todo, considerando que, durante el 2017 , más del $65 \%$ de los casos de enfermedad invasiva de aislamientos obtenidos de 26 hospitales del país correspondieron a serotipos no incluidos en la vacuna en uso. Es importante identificar los serotipos que empiezan a ser más prevalentes, y particularmente cuáles de ellos causarán la enfermedad más grave ${ }^{3,8,9}$. GIVEBPVac $^{12}$ ha mantenido un monitoreo de casos de enfermedad invasiva en una red de hospitales en México, cuyas proyecciones permitirán orientar, con mayor direccionalidad, hacia el tipo de vacuna con una mejor cobertura de acuerdo con los serotipos prevalentes en la actualidad. El reto es conocer si las nuevas formulaciones de vacunas conjugadas próximas a liberarse podrán logar una adecuada cobertura.

\section{Responsabilidades éticas}

Protección de personas y animales. Los autores declaran que para esta investigación no se han realizado experimentos en seres humanos ni en animales.

Confidencialidad de los datos. Los autores declaran que han seguido los protocolos de su centro de trabajo sobre la publicación de datos de pacientes.

Derecho a la privacidad y consentimiento informado. Los autores han obtenido el consentimiento informado de los pacientes o individuos referidos en el artículo. Este documento obra en poder del autor de correspondencia.

\section{Conflicto de intereses}

Los autores declaran no tener ningún conflicto de intereses.

\section{Financiamiento}

Ninguno.

\section{Bibliografía}

1. Echániz-Avilés IG, Solórzano-Santos F. Meeting the challenge: prevention of pneumococcal disease with conjugate vaccines. Salud Publica Mex. 2001;43:352-67.

2. Black S, Shinefield H, Fireman B, Lewis E, Ray P, Hansen JR, et al. Efficacy, safety and immunogenicity of heptavalent pneumococcal conjugate vaccine in children. Northern California Kaiser Permanente Vaccine Study Center Group. Pediatr Infect Dis J. 2000;19:187-95.

3. Echaniz-Aviles G, Soto-Nogueron A, Miranda-Novales G, Carnalla-Barajas MN, Velazquez-Meza ME, Solórzano-Santos F, SIREVA NetworkMexico, et al. Streptococcus pneumoniae serotypes identified in Mexican children with invasive disease before and after the introduction of PCV7 (1993-2012). Arch Med Res. 2015;46:149-53.

4. Lewnard JA, Hanage WP. Making sense of differences in pneumococcal serotype replacement. Lancet Infect Dis. 2019;19:PE213-20.

5. Feikin DR, Kagucia EW, Loo JD, Link-Gelles R, Puhan MA, Cherian T, et al. Serotype-specific changes in invasive pneumococcal disease after pneumococcal conjugate vaccine introduction: a pooled analysis of multiple surveillance sites. PLoS Medicine. 2013;10:e1001517.

6. Balsells E, Guillot L, Nair H, Kyaw MH. Serotype distribution of Streptococcus pneumoniae causing invasive disease in children in the post-PCV era: a systematic review and meta-analysis. PLoS One. 2017;12:e0177113.

7. Shen M, Yao R, Yue H, Zhang J, Chen M, Zhang W, et al. Serotype prevalence and antibiotic susceptibility patterns of pneumococcal isolates in Zunyi city, China. Saudi Med J. 2017;38:1243-9.

8. Carnalla-Barajas MN, Soto-Noguerón A, Sánchez-Alemán MA, Solórzano-Santos F, Velazquez-Meza ME, Echániz-Aviles G, Grupo SIREVA-Mexico, et al. Changing trends in serotypes of $S$. pneumoniae isolates causing invasive and non-invasive diseases in unvaccinated population in Mexico (2000-2014). Int J Infect Dis. 2017;58:1-7.

9. Soto-Noguerón A, Carnalla-Barajas MN, Solórzano-Santos F, Arrendondo-García JL, Arzate-Barbosa P, Tinoco-Favila JC, et al. Streptococcus pneumoniae as cause of infection in infants less than 60 days 
Bol Med Hosp Infant Mex. 2019;76

of age: serotypes and antimicrobial susceptibility. Int J Infect Dis. 2016; 42:69-73.

10. Morales M, Ludwig G, Ercibengoa M, Esteva C, Sanchez-Encinales V Alonso M, et al. Changes in the serotype distribution of Streptococcus pneumoniae causing otitis media after PCV13 introduction in Spain PLoS One. 2018;13:e0209048.

11. Yildirim I, Little BA, Finkelstein J, Lee G, Hanage WP, Shea K, The Massachusetts Dept. of Public Health, et al. Surveillance of pneumococcal colonization and invasive pneumococcal disease reveals shift in prevalent carriage serotypes in Massachusetts' children to relatively low invasiveness. Vaccine. 2017:35:4002-9.

12. Instituto Nacional de Salud Pública [Internet]. GIVEBPVac (Grupo Interdisciplinario para el Estudio de Enfermedades Bacterianas Prevenibles por Vacunación). Datos por sexo y por grupos de edad de los aislamientos de Streptococcus penumoniae, Haemophlilus influenzae, Staphylococcus aureus y Neisseria meningitidis en procesos infecciosos; 2017. Disponible en: https://www.insp.mx/lineas-de-investigacion/medicamentos-en-salud-publica/sireva.html.

13. Lee JK, Yun KW, Choi EH, Kim SJ, Lee SY, Lee HJ. Changes in the serotype distribution among antibiotic resistant carriage Streptococcus pneumoniae isolates in children after the introduction of the extended-valency pneumococcal conjugate vaccine. J Korean Med Sci. 2017;32:1431-9.

14. Gaviria Agudelo C, Jordan Villegas A, Garcia C, McCracken Jr G. The effect of 13-valent pneumococcal conjugate vaccine on the serotype distribution and antibiotic resistance profiles in children with invasive pneumococcal disease. J Pediatric Infect Dis Soc. 2017;6:253-9. 